\title{
The use of kurtosis de-noising for EEG analysis of patients suffering from Alzheimer's disease ${ }^{1}$
}

\author{
Gang Wang ${ }^{\mathrm{a}}$, Simon J. Shepherd ${ }^{\mathrm{b}}$, Clive B. Beggs ${ }^{\mathrm{b}}$, Nini Rao ${ }^{\mathrm{a}}$ and Ying Zhang, ${ }^{\mathrm{a}, *}$ \\ ${ }^{a}$ University of Electronic Science and Technology of China, 611731, Chengdu, China \\ ${ }^{b}$ Medical Biophysics Group, School of Engineering, Design and Technology University of Bradford, \\ BD7 1DP, Bradford, UK
}

\begin{abstract}
The use of electroencephalograms (EEGs) to diagnose and analyses Alzheimer's disease (AD) has received much attention in recent years. The sample entropy (SE) has been widely applied to the diagnosis of AD. In our study, nine EEGs from 21 scalp electrodes in $3 \mathrm{AD}$ patients and 9 EEGs from 3 age-matched controls are recorded. The calculations show that the kurtoses of the AD patients' EEG are positive and much higher than that of the controls. This finding encourages us to introduce a kurtosis-based de-noising method. The 21-electrode EEG is first decomposed using independent component analysis (ICA), and second sort them using their kurtoses in ascending order. Finally, the subspace of EEG signal using back projection of only the last five components is reconstructed. SE will be calculated after the above de-noising preprocess. The classifications show that this method can significantly improve the accuracy of SE-based diagnosis. The kurtosis analysis of EEG may contribute to increasing the understanding of brain dysfunction in $\mathrm{AD}$ in a statistical way.
\end{abstract}

Keywords: Electroencephalogram (EEG), Alzheimer's disease (AD), kurtosis, sample entropy (SE)

\section{Introduction}

Alzheimer's disease (AD) is the most common cause of dementia in people over the age of 65 years [1-4]. It is a neurodegenerative disease characterized by progressive cognitive deterioration, decline in the ability to perform daily activities, and behavioral changes. These symptoms are accompanied by histological changes in the brain, characterized by the formation of extracellular amyloid plaques and the intracellular deposition of neurofibrillary tangles [5]. AD can be difficult to diagnose, particularly in the early stages, and definite diagnosis is only possible by necropsy [6]. Consequently, while the person is alive, it is only possible to make a diagnosis of 'possible' or 'probable' AD. It was shown in [1] that clinical diagnosis of possible $\mathrm{AD}$ has accuracy rates of about $50 \%$. The low diagnosis rate

\footnotetext{
${ }^{1}$ This work was supported by National Science Foundation of China under Grants 61371182 and 41301459, and the State Scholarship Fund of China.

* Address for correspondence: Ying Zhang, University of Electronic Science and Technology of China, 611731, Chengdu, China. Tel.: +86 28 61831571; Fax: +86 28 61830633; E-mail: zhangyingxf@uestc.edu.cn.
}

0959-2989/15/\$35.00 @ 2015 - IOS Press and the authors. 
makes it urgent to develop methodologies for accurately diagnosing AD in patients, particularly during the early stages when there is the best chance to treat the symptoms of the disease.

Although a definitive diagnosis can be made after death, in recent decades, the electroencephalogram (EEG), neuroimaging diagnostic tools such as PET, SPECT, and AMRI have proved useful in aiding the diagnosis of AD. This paper is focused on EEG. Indeed, several studies have associated changes in quantitative spectral electroencephalogram (q-EEG) with cognitive decline in patients with $\mathrm{AD}$ [7]. $\mathrm{AD}$ patients are characterized by EEG abnormalities which shift the power spectrum to the lower frequencies and a decrease in coherence amongst the cortical areas [6], although in the early stages of the disease, EEG plots may exhibit normal frequencies [8]. So although useful, EEG diagnosis can be inconclusive and careful interpretation is required in order to identify AD using this method. Indeed, such is the difficulty of accurately diagnosing AD using EEG that it has led researchers to seek superior methods for analyzing EEG data. To this end, many statistical, signal processing and nonlinear dynamical methods have been used with varying degrees of success. Some of these methods work as preprocessing or de-noising, such as blind source separation (BSS) [9, 10]. Some of these methods work as feature extraction, such as windowed signal power (WP) [10], bipolarity [11], coherence or synchronization $[12,13]$, even-related potentials (ERP)-based feature [14], power frequency (PF) [15, $16]$ and stochastic synchrony $[17,18]$. Other methods work as classifier, such as linear discriminant analysis (LDA) [9], sample entropy (SE) [5, 6, 19-22].

Based on above methods, the authors in [23] conducted different methods of the various combinations as: $\mathrm{PF}+\mathrm{CSP}+\mathrm{LDA}, \mathrm{WP}+\mathrm{CSP}+\mathrm{LDA}, \mathrm{SE}+\mathrm{CSP}+\mathrm{LDA}, \mathrm{PF}+\mathrm{P} 4+\mathrm{LDA}, \mathrm{WP}+\mathrm{P} 4+\mathrm{LDA}, \mathrm{SE}+\mathrm{P} 4+\mathrm{LDA}$, where $\mathrm{P} 4$ denotes one EEG electrode. The performances of these methods are usually influenced by some kind of noise, such as muscle and line noise, and the noise shows different influence in different methods, so one tendency is to develop different de-noising method for different classifier. For example, LDA classifier is utilized in [9], and the AMUSE-BSS is proposed to help de-noise, where the components with the lowest linear predictability are removed from raw data. This paper is focused on how to help sample entropy de-noise.

Sample entropy (i.e. estimation of signal complexity), which was first proposed as a method for investigating the dynamics of heart rate and other time series [24, 25], has been widely applied to the diagnosis of $\mathrm{AD}[5,19,20]$. In particular, Abasolo et al. [5, 19] demonstrated that SE could be used to discriminate $\mathrm{AD}$ patients from normal controls. This approach is based on the hypothesis that EEG plots of $\mathrm{AD}$ patients exhibit greater regularity than those of age-matched controls. Thus, the EEG entropy exhibited by AD patients should be different from that exhibited by age-matched controls. More recent research suggests that the SE approach may be limited due to signal noise [23]. Then it can be hypothesized that SE might work better in an $\mathrm{AD}$ application if suitable de-noising measures were first undertaken.

While a number of de-noising algorithms have been applied to EEG data from AD patients, such as the most relevant components algorithm based on AMUSE $[9,26]$ and common spatial patterns (CSP) $[23,27,28]$, these appear not to be suitable preprocessing tools of SE. From a nonlinear dynamical viewpoint, the regularity of the EEG data collected from $\mathrm{AD}$ patients is greater than that from control patients.

When viewed from a statistical signal processing standpoint, the EEG data from controls are so irregular that they can be regarded as near-Gaussian signals, while that from AD patients are so regular that they can be regarded as far-Gaussian signals. Therefore, EEG signals can be evaluated in terms of their level of "Gaussianity", which is quantified by their kurtosis value (which is a measure of nonGaussianity) $[29,30]$. The lower the absolute value of kurtosis, the more near-Gaussian is the signal, and vice versa. If it is applied to EEG data from AD patients, the non-Gaussian components can be 
regarded as useful signals while the near-Gaussian component can be regarded as useless noise. Note that the kurtosis is the measure of "Gaussianity". This motivates us to identify AD sufferers from EEG plots using the kurtosis to help denoising.

In this paper, a modified sample entropy (SE) approach was used to evaluate sample EEG plots and found this new methodology to be able to distinguish between patients with and without AD with great accuracy. The 21-electrode EEG data were first decomposed using an independent component analysis (ICA) method [31-33], and then the data were sorted by their kurtosis value in descending order. Finally, the subspace of EEG signal was reconstructed using back projection of only the first several components with far-Gaussian property. After the de-noising preprocess, the SE approach was applied to the EEG data.

Table 1

Kurtosis of EEGs of controls for all electrodes

\begin{tabular}{|c|c|c|c|c|c|c|c|c|c|}
\hline kurtosis & EEG1 & EEG2 & EEG3 & EEG4 & EEG5 & EEG6 & EEG7 & EEG8 & EEG9 \\
\hline Fp1 & 0.6828 & 0.0985 & 0.8014 & 0.0680 & 0.0846 & 0.0425 & 11.4655 & 11.7081 & 16.1772 \\
\hline Fp2 & 4.8635 & 9.3026 & 6.1694 & 0.7516 & 2.8487 & 0.7829 & 0.8904 & 0.2357 & 0.9938 \\
\hline F3 & 4.5403 & 8.8131 & 4.4459 & 0.5435 & 1.2553 & 0.5818 & 2.7173 & 1.6430 & 1.3271 \\
\hline F4 & 1.9128 & 7.6254 & 4.2684 & 0.6340 & 1.3354 & 2.2668 & 4.2738 & 14.1135 & 3.3162 \\
\hline $\mathrm{C} 3$ & 2.1537 & 6.2253 & 7.1602 & 0.0848 & 0.1262 & 1.4552 & 7.5946 & 35.9307 & 10.9414 \\
\hline $\mathrm{C} 4$ & 2.9638 & 1.9331 & 4.2397 & 0.0494 & 0.1701 & 7.6063 & 3.6163 & 10.3625 & 5.3654 \\
\hline P3 & 1.9374 & 6.0670 & 8.4925 & 0.1752 & 0.4533 & 0.7876 & 1.4478 & 0.8565 & 2.4335 \\
\hline $\mathrm{P} 4$ & 2.2931 & 2.9128 & 4.6395 & 0.8932 & 2.8330 & 2.3080 & 0.4756 & 0.2502 & 0.6900 \\
\hline $\mathrm{O} 1$ & 1.3404 & 1.2470 & 2.4450 & 1.3907 & 1.0204 & 1.2129 & 2.7040 & 0.7212 & 2.8280 \\
\hline $\mathrm{O} 2$ & 21.6754 & 5.0561 & 4.0545 & 0.2709 & 0.3598 & 0.2957 & 5.8991 & 4.3587 & 5.7090 \\
\hline F7 & 1.9853 & 0.5176 & 0.7739 & 0.4236 & 0.3373 & 0.4563 & 2.6497 & 1.9802 & 2.1083 \\
\hline F8 & 0.3838 & 0.2931 & 0.1030 & 0.2490 & 0.1514 & 0.2770 & 0.6528 & 1.2226 & 1.0271 \\
\hline $\mathrm{T} 7$ & 2.3961 & 0.5298 & 2.1150 & 1.9071 & 0.8121 & 0.6395 & 0.7568 & 0.7805 & 0.6437 \\
\hline $\mathrm{T} 8$ & 4.0216 & 5.6961 & 4.0315 & 0.2212 & 0.1878 & 3.4055 & 0.3872 & 0.2411 & 0.4400 \\
\hline P7 & 0.9496 & 0.1995 & 0.3442 & 0.0805 & 0.2841 & 0.0174 & 0.3416 & 0.6345 & 0.6151 \\
\hline P8 & 3.5042 & 7.4554 & 9.2163 & 0.8296 & -0.0656 & 0.1233 & 2.1008 & 17.0041 & 4.9070 \\
\hline $\mathrm{FZ}$ & 0.7913 & 1.9702 & 1.0507 & 0.2636 & 0.3850 & 0.5184 & 1.8433 & 3.0732 & 2.3913 \\
\hline $\mathrm{CZ}$ & 0.2943 & 0.1514 & 2.3762 & 0.0041 & -0.0321 & -0.1079 & 2.0886 & 1.5938 & 4.5597 \\
\hline $\mathrm{PZ}$ & 12.3836 & 0.3773 & 221.0881 & 0.4186 & 0.2591 & 0.6007 & 0.6468 & 0.3631 & 1.1326 \\
\hline FPZ & 1.7342 & 0.7820 & 15.3238 & 0.1985 & 0.1636 & 0.2011 & 0.2548 & 0.3279 & 0.5295 \\
\hline $\mathrm{OZ}$ & 1.0717 & 0.8227 & 5.2999 & -0.3547 & -0.2409 & -0.1155 & 1.2644 & 1.2625 & 1.2614 \\
\hline Means & 3.8163 & 2.9518 & 6.2992 & 0.3969 & 0.8625 & 1.6798 & 1.5715 & 2.3819 & 2.2331 \\
\hline
\end{tabular}




\section{Method}

\subsection{EEG data and its kurtosis}

In order to test the algorithm, the EEG data were recorded from three times from $3 \mathrm{AD}$ patients and three age-matched controls, respectively. EEGs were collected from the 21 scalp loci of the international 10-20 system (electrode Fp1, Fp2, F3, F4, C3, C4, P3, P4, O1, O2, F7, F8, T7, T8, P7, P8 FZ, $\mathrm{CZ}, \mathrm{PZ}, \mathrm{FPZ}$ and OZ). A specialist physician to check for eye movement and other artifacts inspected all EEGs. Since the EEG data are not stationary, we divided the EEG data from one person into three parts and each part contains 10000 data points where the sample rate was $100 \mathrm{~Hz}$. Thus, the 9 EEGs data from $\mathrm{AD}$ patients and 9 EEGs data from controls were obtained, and each EEG data have the size of $21 \times 10000$.

Kurtosis measures the relative flatness of a distribution. Distributions with positive kurtosis are termed "super-Gaussian", while those with negative kurtosis are "sub-Gaussian". If $\kappa_{i}$ denotes the kurtosis of the output $y_{i}(i=1,2, \ldots, n), M_{4 i}$ the 4 th moment of $y_{i}$, and $M_{2 i}$ the 2 nd moment of $y_{i}$, then the kurtosis can be estimated via the following algorithm [34]:

$$
\begin{aligned}
& \kappa_{i}^{+}=\frac{M_{4 i}^{+}}{M_{2 i}^{+}}-3 \\
& M_{4 i}^{+}=\delta M_{4 i}+(1-\delta)\left|y_{i}\right|^{4} \\
& M_{2 i}^{+}=\delta M_{2 i}+(1-\delta)\left|y_{i}\right|^{2}
\end{aligned}
$$

where $\delta(0<<\delta<1)$ is a constant.

Then the kurtosis of the data in each electrode for controls is shown in Table 1 and that of AD patients is shown in Table 2. It can be seen from the two tables that most of the kurtoses of EEGs of AD patients are higher than that of controls. This motivates us to introduce kurtosis-based method to help to de-noise the EEG data.

\subsection{ICA for EEG de-noising}

Implicit in the concept of de-noising is the expectation that within a noisy signal exists hidden components that impart core information relating to the complex signal under consideration. With respect to EEG data, these components are more sensitive in AD patients than in others, and thus can be considered as useful 'signals'. By comparison, the other components of the EEG data are 'noise' and thus can be considered 'useless signals.' Thus by de-noising, or improving the signal-to-noise ratio (SNR) by filtering off the 'noise,' it should be possible to enhance the performance of subsequent feature extraction and data classification. With regard about this, ICA algorithms [26] can be used for the purpose of filtering. ICA is used to extract independent source signals from their linear mixtures without knowing the mixing parameters. It is a statistical method and has attracted considerable attention amongst the signal processing and neural network community. When applying ICA to EEG analysis, if $\boldsymbol{x}=\left(x_{1}, x_{2}, \ldots, x_{\mathrm{m}}\right)^{\mathrm{T}}$ denotes an $m$-dimension observation vector, and $\boldsymbol{s}=\left(s_{1}, s_{2}, \ldots, s_{\mathrm{n}}\right)^{\mathrm{T}}$ an $n$-dimension hidden independent components (ICs) - some of which may be evident in AD patients, then the linear relationship is given by

$$
x=A s
$$


where $\boldsymbol{A}$ is an $m \times n$ unknown matrix, called the mixing matrix. Here it is assumed that $m$ is equal to $n$, and $\boldsymbol{A}$ is full rank. The basic problem of ICA is then to conduct an $n \times n$ full-rank separating matrix $\boldsymbol{B}$ given just the observation sequence with the restriction that source elements $s_{i}$ are non-Gaussian independent components. Thus the output vector

$$
\boldsymbol{y}=\boldsymbol{B} \boldsymbol{x}, \boldsymbol{y}=\left(y_{1}, y_{2}, \ldots, y_{n}\right)^{T}
$$

provides the estimates of the sources signals. Due to two kinds of inherent indeterminacies in Eq. (1), it is impossible to recover the source signals in exact amplitude or in exact order [26,30]. Without loss of generality, the source signals are assumed to have unit variance with any scaling factors absorbed into the mixing matrix $\boldsymbol{A}$. In other words, when a perfect ICA is performed, the global transfer matrix $\boldsymbol{C}=\boldsymbol{B} \boldsymbol{A}$ should be an $n \times n$ generalized permutation matrix. Many algorithms designed for ICA have been proposed, including those employing high order statistics (HOS), such as fast ICA [30] and flexible ICA [34], and those employing second-order statistics (SOS), such as second order blind identifi

Table 2

Kurtosis of EEGs of AD patients for all electrodes

\begin{tabular}{l|lllllllll}
\hline kurtosis & EEG1 & EEG2 & EEG3 & EEG4 & EEG5 & EEG6 & EEG7 & EEG8 & EEG9 \\
\hline Fp1 & 25.4143 & 0.3365 & 25.3954 & 0.0950 & 0.0332 & -0.0090 & 2.6302 & 0.3716 & 0.8848 \\
Fp2 & 9.9425 & 2.9189 & 15.3509 & 20.8243 & 13.7805 & 13.2179 & 6.8940 & 8.3364 & 6.2143 \\
F3 & 11.2298 & 5.6626 & 3.5790 & 6.4451 & 174.1147 & 6.9603 & 24.6065 & 91.9136 & 15.6406 \\
F4 & 2.3398 & 1.3652 & 14.6675 & 0.4203 & 586.1999 & 0.0696 & 5.5400 & 8.5658 & 2.8525 \\
C3 & 15.2431 & 1.5070 & 710.9700 & 5.3769 & 18.8216 & 1.8099 & 5.9622 & 3.1621 & 3.9944 \\
C4 & 1.7673 & 0.1861 & 1.0985 & 1.1406 & 0.9838 & 2.4726 & 1.3227 & 0.9336 & 0.6748 \\
P3 & 11.5065 & 1.1540 & 3.0467 & 0.8154 & 9.7243 & 2.3595 & 3.0037 & 7.9806 & 3.5677 \\
P4 & 4.5861 & 2.7389 & 6.3293 & 4.8936 & 7.8389 & 4.2088 & 2.5446 & 11.4943 & 2.8517 \\
O1 & 5.2264 & 0.7323 & 22.2497 & 1.1920 & 59.4170 & 5.0022 & 0.9179 & 0.2470 & 0.4685 \\
O2 & 28.4244 & 4.0033 & 21.8968 & 0.6144 & 5.1121 & 0.4355 & 1.6995 & 1.0665 & 1.6507 \\
F7 & 11.5029 & 0.3558 & 10.9458 & 0.3430 & 0.3652 & 2.7869 & 3.5040 & 3.1700 & 12.3627 \\
F8 & 0.4262 & 0.4003 & 0.9675 & 0.1333 & 0.1420 & 12.2024 & 1.3489 & 0.0861 & 0.8716 \\
T7 & 1.0529 & 0.6289 & 2.0512 & 6.7394 & 2.1853 & 25.8189 & 1.6907 & 1.4805 & 1.6151 \\
T8 & 2.7040 & 1.3900 & 23.7984 & 8.9052 & 3.1716 & 21.9267 & 2.2837 & 0.6258 & 0.7632 \\
P7 & 4.8916 & 2.7803 & 22.9866 & 27.2410 & 21.9844 & 56.3523 & 0.5131 & 0.2897 & 0.3514 \\
P8 & 27.4883 & 0.3791 & 28.5912 & 1.0208 & 0.0693 & 0.0041 & 23.7412 & 9.3029 & 4.6342 \\
FZ & 22.5844 & 1.3900 & 36.6615 & 1.3416 & -0.0949 & -0.0784 & 3.3059 & 0.8454 & 0.5356 \\
CZ & 2.7989 & 1.2735 & 51.6157 & 2.2172 & 1.5684 & 19.0611 & 10.6106 & 1.7113 & 1.5719 \\
PZ & 5.5300 & 0.1359 & 11.0895 & 1.4354 & 10.4328 & 6.5352 & 1.9583 & 1.1752 & 0.3318 \\
FPZ & 15.2469 & 1.1504 & 28.3517 & 0.2694 & 1.2227 & 29.6515 & 4.7314 & 1.5520 & 0.7005 \\
OZ & 28.9541 & 0.1200 & 15.2411 & 0.1499 & -0.0240 & -0.0222 & 4.6474 & 2.3591 & 1.2358 \\
\cline { 2 - 8 } Means & 11.0842 & 2.4350 & 30.3846 & 2.3811 & 136.8885 & 2.9083 & 3.2937 & 3.4279 & 3.1594 \\
\hline & & & & & & & & & \\
\hline
\end{tabular}


(SOBI) [35], the AMUSE algorithm [26] and mean square cross prediction error (MSCPE) algorithm $[8,31]$. Generally speaking, the HOS algorithms are more time-consuming than SOS, therefore in order to save time AMUSE, one of the SOS algorithms, was used in the experiments below.

AMUSE algorithm is similar to PCA or singular vale decomposition (SVD). AMUSE utilizes PCA/SVD two times. In the first step, PCA/SVD can be applied for whitening. Whitening removes the second-order dependence among the observations $\boldsymbol{x}$. If the autocorrelation matrix $\boldsymbol{R}_{\boldsymbol{x}}=E\left\{\boldsymbol{x} \boldsymbol{x}^{\mathrm{T}}\right\}$ has the SVD, $\boldsymbol{R}_{\boldsymbol{x}}=\boldsymbol{P} \boldsymbol{D} \boldsymbol{P}^{T}$, where $\boldsymbol{D}=\operatorname{diag}\left(d_{1}, d_{2}, \ldots, d_{n}\right)$ is a diagonal matrix of corresponding eigenvalues, the suitable whitening matrix will be $\boldsymbol{W}=\boldsymbol{P} \boldsymbol{D}^{-1 / 2} \boldsymbol{P}^{T}$. Then $\widetilde{x}=\boldsymbol{W} \boldsymbol{x}$ is a standardized vector satisfying $E_{\left\{\widetilde{\boldsymbol{x}}^{T}\right\}=\boldsymbol{I}}$. In the second step, SVD/PCA is utilized as

$$
\boldsymbol{R}\left(\boldsymbol{x}_{1}\right)=\boldsymbol{E}\left\{\tilde{\boldsymbol{x}}(k) \tilde{\boldsymbol{x}}^{T}(k-1)\right\}=\boldsymbol{U} \boldsymbol{\Sigma} \boldsymbol{V}^{T}
$$

where $\boldsymbol{\Sigma}$ is diagonal matrix, and $\boldsymbol{U}, \boldsymbol{V}$ are orthogonal matrices. Then, a global separating matrix is estimated as $\boldsymbol{B}=\boldsymbol{U}^{T} \boldsymbol{W}[26]$

$$
\begin{aligned}
& \boldsymbol{y}=\boldsymbol{B} \boldsymbol{x}=\boldsymbol{U}^{T} \boldsymbol{W} \boldsymbol{x} \\
& \boldsymbol{y}=\left(y_{1}, y_{2}, \ldots, y_{n}\right)^{T}
\end{aligned}
$$

where the kurtosis of $y_{i}$ is sort as follows for simplicity:

$$
\kappa_{1}>\kappa_{2}>\cdots>\kappa_{n}
$$

As for the separated signal space, it can be divided into near-Gaussian and far-Gaussian subspaces according to the kurtosis of separated signals. Denote by $\boldsymbol{y}_{\mathrm{ICA}}$ the subspace spanned by the farGaussian components

$$
\boldsymbol{y}_{\text {ICA }}=\left(y_{1}, y_{2}, \ldots, y_{d}\right)^{T}
$$

where $d$ is the number of the far Gaussian components. Then the near-Gaussian noise can be filtered by back projecting observation $\boldsymbol{x}$ onto the far-Gaussian subspace

$$
\boldsymbol{x}_{I C A}=\boldsymbol{y}_{I C A}^{T} \boldsymbol{y}_{I C A} \boldsymbol{x}
$$

In this experiment, the far-Gaussian subspace containing five components with highest positive kurtosis has been selected:

$$
\begin{aligned}
& m=n=21 \\
& d=5
\end{aligned}
$$

As for the selection of the number of the far Gaussian components $(d)$, when $d$ is less than two or more than eight, worse performance will be achieved than in the case of raw data. When $d$ is ranged from four to six, the results of classification will be better. In this paper, the number of the far Gaussian components is adopt as $d=5$. 


\subsection{Calculation of $S E$}

In the detection and diagnosis of $\mathrm{AD}$, the nonlinear characteristics of the EEG time series has been used for some time, such as the correlation dimension (D2) of the EEG time series [36], the first Lyapunov exponent (L1) to distinguish between probable AD and control subjects [20] and SE. However, SE can work well instead of D2 and L1. This point has been proven by [5] and [19]. Moreover, the results of [5] indicate that $\mathrm{SE}$ is a useful feature for discriminating between $\mathrm{AD}$ subjects and controls.

Formally, given $N$ data points form a time series $\boldsymbol{x}(n)=\boldsymbol{x}(1), \boldsymbol{x}(2), \cdots, \boldsymbol{x}(N)$, the basic idea of SE is to approximate the following statistic $[5,23]$ :

$$
S E(m, r)=-\ln \left[\frac{\operatorname{Pr}\left(d\left[x_{m+1}(i), x_{m+1}(j)\right] \leq r\right)}{\operatorname{Pr}\left(d\left[x_{m}(i), x_{m}(j)\right] \leq r\right)}\right]
$$

where $x_{m}(i) \in R^{m}$ is the vector extracted from the time series $\boldsymbol{x}(t)$ as follows:

$$
\boldsymbol{x}_{m}(i)=[x(i), x(i+1), \cdots, x(i+m-1)]
$$

and $d\left[x_{m}(i), x_{m}(j)\right]$ is defined as:

$$
d\left[x_{m}(i), x_{m}(j)\right]=\max _{k=[1, m]}(|x(i+k-1)-x(j+k-1)|)
$$

In practice, the probability values in expression (10) were approximated by averaging over the entire time series. Because of the nonlinear character of EEG signal, SE can be utilized as a powerful tool to measure the regularity of AD patients' EEG. Furthermore, The Matlab tools for calculating sample entropy provided at http://www.physionet.org/physiotools/sampen/matlab/ are used in the experiments. The calculation of Eq. (10) including the approximation of the probability values are performed through the Matlab codes in the above website.

\subsection{Testing for difference between two means}

It is frequently in biology that analysis is undertaken of two populations whose distributions overlap, and statistics is used to determine if the populations are significantly different from each other. In situations like this useful technique that can be applied is the t-test, which can be used to evaluate the statistical difference between $\mathrm{AD}$ patients and the controls, after the SE values have first been obtained from the EEG signals.

If $Z 1$ denotes the set of SE values from one electrode in $\mathrm{AD}$ patients and $\mathrm{Z} 2$ denotes the set of SE values from the controls, the null hypothesis in this case will be: "Z1 and Z2 are equal on average". The t-test therefore allows us to assign a probability level to the likelihood that the null hypothesis is true. The calculation is straightforward, requiring us to calculate both the mean of each set and a measure of the variation of each mean. Here is the formula for calculating t-value 


$$
\begin{aligned}
& t_{\text {value }}=\frac{\operatorname{mean}(Z 1)-\operatorname{mean}(Z 2)}{z_{p}\left(1 / n_{1}+1 / n_{2}\right)^{0.5}} \\
& z_{p}^{2}=\frac{\left[\left(n_{1}-1\right) \operatorname{Var}(Z 1)+\left(n_{2}-1\right) \operatorname{Var}(Z 2)\right]}{\left(n_{1}+n_{2}-1\right)}
\end{aligned}
$$

where operator mean() denotes the means of the sets, $\operatorname{Var}()$ denotes the sample variances of the two groups, and $n_{1}$ and $n_{2}$ are the sample sizes of each set.

After getting the $t$-value, the $p$-value can be further calculated. In the experiment, the threshold of $p$ value as [5], i.e. 0.01 , was selected. If the $p$-value was lower than $0.01 \mathrm{ct}$ the null hypothesis of no difference would be rejected, and infer that the differences between the mean values were considered significant. The calculation of the $p$-value, refer to as the website: $\mathrm{http}: / / \mathrm{www}$.danielsoper.com/statcalc/calc08.aspx, where the degrees of freedom for our experiments are 16 ( 9 samples for $\mathrm{AD}+9$ samples for controls - 2).

Combining the kurtosis, AMUSE, SE algorithm and $t$-value test, the new EEG analysis method can be summarized as follows:

Step 1: Each EEG expressed as $\boldsymbol{x}$ was decomposed into 21 independent components by AMUSE algorithm using Eq. (5). (6).

Step 2: Calculate the kurtoses of the separated components, and sort them in descending order as Eq.

Table 3

Average kurtosis of EEGs for $\mathrm{AD}$ patients and controls for all channels

\begin{tabular}{l|llllll}
\hline & \multicolumn{2}{|l}{ AD Patients } & Controls & \multicolumn{2}{l}{ Statistical Analysis } \\
& Mean & STD & Mean & STD & t_value & P_value \\
\hline Fp1 & 3.074 & 5.1475 & 2.088 & 3.0492 & 0.4945 & 0.628 \\
Fp2 & 20.40 & 22.71 & 3.196 & 2.478 & 2.259 & 0.0382 \\
F3 & 72.44 & 174.7 & 3.661 & 2.695 & 1.181 & 0.255 \\
F4 & 149.5 & 431.9 & 5.480 & 4.537 & 0.9998 & 0.332 \\
C3 & 66.56 & 139.3 & 4.217 & 4.093 & 1.342 & 0.198 \\
C4 & 5.949 & 5.442 & 5.957 & 5.127 & 0.0031 & 0.998 \\
P3 & 9.058 & 17.00 & 3.039 & 3.806 & 1.037 & 0.315 \\
P4 & 6.579 & 8.009 & 3.575 & 3.536 & 1.029 & 0.319 \\
O1 & 18.19 & 43.26 & 1.136 & 0.7301 & 1.182 & 0.254 \\
O2 & 6.755 & 9.679 & 2.469 & 1.941 & 1.302 & 0.211 \\
F7 & 4.310 & 5.629 & 0.827 & 0.7421 & 1.840 & 0.0843 \\
F8 & 4.680 & 9.663 & 0.4192 & 0.3787 & 1.321 & 0.205 \\
T7 & 3.531 & 4.816 & 0.4702 & 0.368 & 1.901 & 0.0755 \\
T8 & 7.163 & 29.25 & 0.9911 & 1.151 & 1.051 & 0.309 \\
P7 & 39.41 & 110.8 & 0.6218 & 0.6416 & 1.050 & 0.309 \\
P8 & 7.163 & 1.516 & 1.490 & 11.28 & 1.469 & 0.161 \\
FZ & 8.009 & 17.33 & 0.8348 & 0.5256 & 1.241 & 0.232 \\
CZ & 5.578 & 6.669 & 10.51 & 0.5327 & 2.273 & 0.0371 \\
PZ & 7.240 & 16.23 & 9.192 & 18.24 & 0.2399 & 0.813 \\
FPZ & 3.578 & 3.917 & 1.014 & 1.760 & 1.791 & 0.0923 \\
OZ & 4.049 & 7.718 & 0.5978 & 0.5997 & 1.337 & 0.200 \\
\hline & & & & & &
\end{tabular}


Step 3: Select the first five components for the far-Gaussian subspace ${ }^{y_{I C A}}$ as Eq. (7), project the EEG $x$ onto this subspace as Eq. (8), and obtain $\boldsymbol{x}_{I C A}$.

Step 4: Calculate the SE of the 21 electrodes $\left(\boldsymbol{x}_{I C A}\right)$.

Step 5: Calculate the p-value between two means

\section{Experiments}

The kurtoses for the 21 electrodes Fp1, Fp2, F3, F4, C3, C4, P3, P4, O1, O2, F7, F8, T7, T8, P7, P8 FZ, CZ, PZ, FPZ and OZ were first estimated. Secondly, the SE $(\mathrm{m}=1, \mathrm{r}=0.25$ times the standard deviation of the data) for all 21 electrode under different conditions (i.e. without de-noising, relevant denoising and kurtosis-based de-noising) were calculated. Finally, the receiver operating characteristic (ROC) curves for the different conditions were plotted.

\subsection{The kurtoses of the EEG from AD patients and controls}

Table 3 summarized the average kurtosis of EEGs for the AD patients and controls for all channels. The mean values of almost all electrodes except $\mathrm{C} 4$ and $\mathrm{PZ}$ are much higher than that of normal controls. However, the standard deviation (STD) of AD EEG's kurtosis is very high, and none of the $p$ values in 21 electrodes are lower than 0.01 . Thus, their mean values might be not so significantly dif-

Table 4

Average SE $(m=1, r=0.25)$ of EEGs for AD patients and controls for all channels without denosing

\begin{tabular}{lllllll}
\hline & AD Patients & Controls & & \multicolumn{2}{c}{ Statistical Analysis } \\
& Mean & STD & Mean & STD & $t_{\text {_value }}$ & $p \_$value \\
\hline Fp1 & 1.8583 & 0.0862 & 1.891 & 0.0839 & 0.8164 & 0.426 \\
Fp2 & 1.4919 & 0.1823 & 1.6727 & 0.3242 & 1.4583 & 0.164 \\
F3 & 1.5156 & 0.1956 & 1.6663 & 0.3152 & 1.2188 & 0.241 \\
F4 & 1.7575 & 0.1398 & 1.6395 & 0.2115 & 1.3961 & 0.182 \\
C3 & 1.744 & 0.2131 & 1.6741 & 0.2436 & 0.6484 & 0.526 \\
C4 & 1.8112 & 0.1299 & 1.6651 & 0.2116 & 1.7641 & 0.968 \\
P3 & 1.8373 & 0.0886 & 1.8233 & 0.1371 & 0.2562 & 0.801 \\
P4 & 1.7805 & 0.0676 & 1.7914 & 0.1744 & 0.1747 & 0.864 \\
O1 & 1.8558 & 0.1652 & 1.8993 & 0.0353 & 0.7719 & 0.451 \\
O2 & 1.8157 & 0.1516 & 1.8272 & 0.133 & 0.1712 & 0.866 \\
F7 & 1.8551 & 0.1367 & 1.9181 & 0.0259 & 1.3582 & 0.193 \\
F8 & 1.8989 & 0.0823 & 1.9416 & 0.0243 & 1.4951 & 0.154 \\
T7 & 1.8699 & 0.0808 & 1.9388 & 0.0192 & 2.4902 & 0.0241 \\
T8 & 1.884 & 0.0661 & 1.9184 & 0.0581 & 1.1724 & 0.258 \\
P7 & 1.9057 & 0.0661 & 1.9207 & 0.034 & 0.6083 & 0.552 \\
P8 & 1.9197 & 0.2339 & 1.9211 & 0.0325 & 0.0172 & 0.986 \\
FZ & 1.7689 & 0.4489 & 1.9146 & 0.0271 & 0.9719 & 0.346 \\
CZ & 1.7997 & 0.1751 & 1.9306 & 0.0375 & 2.1927 & 0.0435 \\
PZ & 1.8918 & 0.092 & 1.7882 & 0.3886 & 0.7783 & 0.448 \\
FPZ & 1.8678 & 0.1114 & 1.915 & 0.0492 & 1.1625 & 0.262 \\
OZ & 1.8207 & 0.1606 & 1.9269 & 0.0318 & 1.9466 & 0.0693 \\
\hline
\end{tabular}


ferent as to be used to a classification. This shows on the viewpoint of statistical signal processing that the regularity of AD seems more like super-Gaussian than normal controls.

\subsection{SE under different conditions}

Tables 4-6 summarized the average SE values of EEGs for AD patients and controls for all channels under no de-noising, relevant de-noising and kurtosis de-noising conditions, respectively. From the data in these tables, it can be seen that for most of the electrodes $p$-value $>0.05$, with the lowest $p$-value in Table 4 being only 0.0241 (i.e. for electrode T7), and 0.0246 for electrode F3 in Table 5. This indicates that the relevant denoising method made no improvement, and there are no significantly different mean values in all the electrodes for the two methods. If the accuracy for classification using P4 electrode as $[5,23]$ under this circumstance is calculated, the accuracy will be about $67 \%$, which although consistent with that reported in [23] is not a good result. However, as can be seen in Table 7, if T7 from Table 4, or F3 from Table 5 are selected, then the accuracy of the diagnosis improves to around $80 \%$.

Comparison of the data in Table 6 with that in Table 4 and Table 5 reveals that the kurtosis-based de-noising method resulted in a significant improvement in performance. The lowest $p$-value in Table 6 reached 0.000074 , which indicates significant differences between the mean SE values of AD patients and normal controls. Table 7 summarizes the accuracies of different methods, and shows that the accuracy of kurtosis de-noising method is improved from about $80 \%$ to almost $95 \%$.

Table 5

Average SE $(\mathrm{m}=1, \mathrm{r}=0.25)$ of EEGs for AD patients and controls for all channels after relevant denosing [9]

\begin{tabular}{lllllll}
\hline & \multicolumn{2}{c}{ AD Patients } & \multicolumn{2}{c}{ Controls } & \multicolumn{2}{c}{ Statistical Analysis } \\
& Mean & STD & Mean & STD & \multicolumn{1}{c}{$t$ value } & $p$ value \\
\hline Fp1 & 1.8652 & 0.1143 & 1.9118 & 0.0408 & 1.1536 & 0.266 \\
Fp2 & 1.5331 & 0.2352 & 1.7254 & 0.2059 & 1.8458 & 0.0835 \\
F3 & 1.5028 & 0.1801 & 1.7221 & 0.1948 & 2.4803 & 0.0246 \\
F4 & 1.7668 & 0.1503 & 1.6690 & 0.1693 & 1.2961 & 0.213 \\
C3 & 1.7199 & 0.1942 & 1.6915 & 0.2564 & 0.2654 & 0.794 \\
C4 & 1.7585 & 0.2253 & 1.6952 & 0.2298 & 0.5904 & 0.563 \\
P3 & 1.7667 & 0.1202 & 1.8294 & 0.1133 & 1.1396 & 0.271 \\
P4 & 1.7317 & 0.1117 & 1.7979 & 0.1376 & 1.1203 & 0.279 \\
O1 & 1.8591 & 0.1791 & 1.8691 & 0.0915 & 0.1490 & 0.883 \\
O2 & 1.8751 & 0.1336 & 1.8255 & 0.1170 & 0.8374 & 0.414 \\
F7 & 1.8839 & 0.1501 & 1.9094 & 0.0567 & 0.4764 & 0.640 \\
F8 & 1.8866 & 0.1489 & 1.9193 & 0.0435 & 0.6326 & 0.536 \\
T7 & 1.8281 & 0.1905 & 1.9200 & 0.0323 & 1.4273 & 0.173 \\
T8 & 1.8864 & 0.0818 & 1.9044 & 0.0472 & 0.5706 & 0.576 \\
P7 & 1.9016 & 0.0908 & 1.8981 & 0.0472 & 0.1018 & 0.920 \\
P8 & 1.9142 & 0.0735 & 1.9289 & 0.0348 & 0.5442 & 0.594 \\
FZ & 1.7463 & 0.4002 & 1.9246 & 0.0324 & 1.3319 & 0.202 \\
CZ & 1.8077 & 0.1723 & 1.9305 & 0.0441 & 2.0709 & 0.549 \\
PZ & 1.8819 & 0.0947 & 1.7842 & 0.3953 & 0.7218 & 0.481 \\
FPZ & 1.8790 & 0.1431 & 1.9149 & 0.0645 & 0.6867 & 0.502 \\
OZ & 1.8603 & 0.2092 & 1.9141 & 0.0652 & 0.7368 & 0.472 \\
\hline
\end{tabular}


Furthermore, the ROC curves were plot for the no de-noising, relevant de-noising and kurtosis denoising methods in Figure 1. ROC curves were obtained by plotting the sensitivity values on the $y$-axis against their equivalent $\{1$-specificity $\}$ values for all the available cut-off points (in this case, the kurtoses or SE values) on the $x$-axis. The sensitivity value represents the percentage of patients with a diagnosis of $\mathrm{AD}$ who are tested as positive, or the true positive rate. The specificity means the proportion of controls correctly recognized, or the true negative rate [5]. Figure 1 reveals that there are no significant differences between the no de-noising method and relevant de-noising method, and that the area under ROC curve of our proposed method (kurtosis de-noising) is much larger than those of the other two methods. Table 7 summarized the corresponding values under ROC curves, where the accuracy of the proposed method is more than $90 \%$.

Generally speaking, the larger the area under ROC curve, the better the performance [5]. With values between 0.90 and 1 , the precision of the diagnostic test is considered to be excellent, good for values between 0.80 and 0.89 , fair for values between 0.70 and 0.79 , poor for values between 0.60 and 0.69 , and bad for values between 0.50 and 0.59 . As a result, the kurtosis-based de-noising method can be considered excellent for electrode F3, the relevant de-noising method can be good for F3, the SE method without de-noising can be good for T7 and poor for P4.

As for the classification, the simple threshold method was adopted. The classifications were depicted in Figure 2, where 'o' denotes normal controls, '*' denotes AD patients, and '-' denotes threshold. The corresponding accuracies were shown in Table 7. Both visual detections in Figure 2 and accuracies in Table 7 show that the proposed method performs better than the others.

Table 6

Average SE $(m=1, r=0.25)$ of EEGs for AD patients and controls for all channels after kurtosis denosing

\begin{tabular}{lllllll}
\hline & \multicolumn{2}{l}{ AD Patients } & Controls & \multicolumn{3}{c}{ Statistical Analysis } \\
& Mean & STD & Mean & STD & $t \_$value & $p$ _value \\
\hline Fp1 & 1.6623 & 0.1788 & 1.8403 & 0.064 & 2.8113 & 0.0125 \\
Fp2 & 1.6371 & 0.1269 & 1.7537 & 0.172 & 1.6375 & 0.121 \\
F3 & 1.5376 & 0.1414 & 1.8096 & 0.0616 & 5.2874 & $\mathbf{0 . 0 0 0 0 7 4}$ \\
F4 & 1.7002 & 0.1198 & 1.6677 & 0.2032 & 0.4138 & 0.685 \\
C3 & 1.7048 & 0.1758 & 1.6915 & 0.249 & 0.1307 & 0.898 \\
C4 & 1.6363 & 0.1991 & 1.6664 & 0.2183 & 0.3061 & 0.763 \\
P3 & 1.7359 & 0.1191 & 1.8161 & 0.1333 & 1.3459 & 0.197 \\
P4 & 1.7037 & 0.1123 & 1.7672 & 0.1386 & 1.0669 & 0.302 \\
O1 & 1.6983 & 0.1412 & 1.8551 & 0.0755 & 2.9385 & $\mathbf{0 . 0 0 9 6 4}$ \\
O2 & 1.7346 & 0.145 & 1.7494 & 0.1522 & 0.2122 & 0.835 \\
F7 & 1.7548 & 0.1159 & 1.7695 & 0.1443 & 0.2382 & 0.915 \\
F8 & 1.6894 & 0.1555 & 1.8018 & 0.1432 & 1.5957 & 0.13 \\
T7 & 1.7833 & 0.1166 & 1.7565 & 0.1973 & 0.3505 & 0.731 \\
T8 & 1.7327 & 0.1563 & 1.8602 & 0.1062 & 2.0242 & 0.06 \\
P7 & 1.6797 & 0.1836 & 1.8269 & 0.094 & 2.14 & 0.0481 \\
P8 & 1.7158 & 0.1305 & 1.8048 & 0.101 & 1.6172 & 0.125 \\
FZ & 1.668 & 0.3549 & 1.8112 & 0.0911 & 1.1731 & 0.258 \\
CZ & 1.7156 & 0.1597 & 1.8157 & 0.1683 & 1.294 & 0.214 \\
PZ & 1.7274 & 0.1247 & 1.6477 & 0.4114 & 0.556 & 0.586 \\
FPZ & 1.7128 & 0.1597 & 1.8566 & 0.0649 & 2.5023 & 0.0236 \\
OZ & 1.719 & 0.1909 & 1.8201 & 0.1257 & 1.3259 & 0.203 \\
\hline
\end{tabular}


Table 7

Accuracy results under three different conditions

\begin{tabular}{lllll}
\hline Method & Electrode & Threshold & Accuracy & Area under ROC curve \\
\hline No de-noising & P4 & 1.800 & $66.7 \%$ & $63.6 \%$ \\
No de-noising & T7 & 1.904 & $83.3 \%$ & $81.5 \%$ \\
Relevant de-noising & F3 & 1.700 & $77.8 \%$ & $80.2 \%$ \\
Kurtosis de-noising & F3 & 1.700 & $94.4 \%$ & $97.5 \%$ \\
\hline
\end{tabular}

Furthermore, the comparisons were done with other de-noising algorithms, ASUME [9] and common spatial patterns (CSP) [27], in Table 8, which shows that the proposed algorithm (Kurtosis denoising) works better than the other two methods.

\section{Conclusions}

In this study, the Gaussianity of the EEG signal from a number of AD patients and normal controls using kurtosis methods were analyzed. The average kurtosis of the EEG signals from the AD patients was found to be much higher than that of the controls. This implies that the EEG signals from individuals with $\mathrm{AD}$ contain different information, which if identified could prove useful in diagnosing the condition. The SE approach is based on the hypothesis that the regularity of EEG signals form AD patients is higher than that of age-matched controls, with the result that the entropy of this group's EEG signals is lower than that of age-matched controls - indicating an abnormal dynamic within the AD group. However, given that the SE approach appears to be limited by signal noise [23], a kurtosisbased de-noising method was used as a preprocessing step of SE for AD diagnosis. The regularity achieved appeared to be super-Gaussian. The statistical analysis was consistent with the nonlinear dynamic study, suggesting that both embodied the property found in the AD EEG signals. Classification of the results verified the efficacy of the approach and it may be that the new method has great potential in the early diagnosis and prediction of AD.

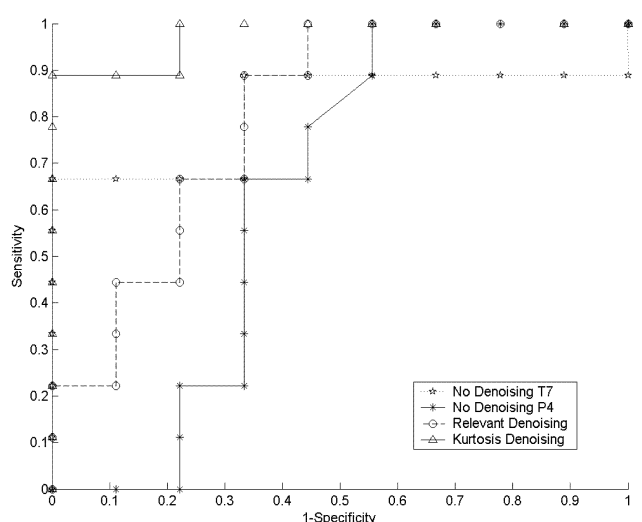

Fig. 1. Receiver operating characteristic (ROC) curves different methods.
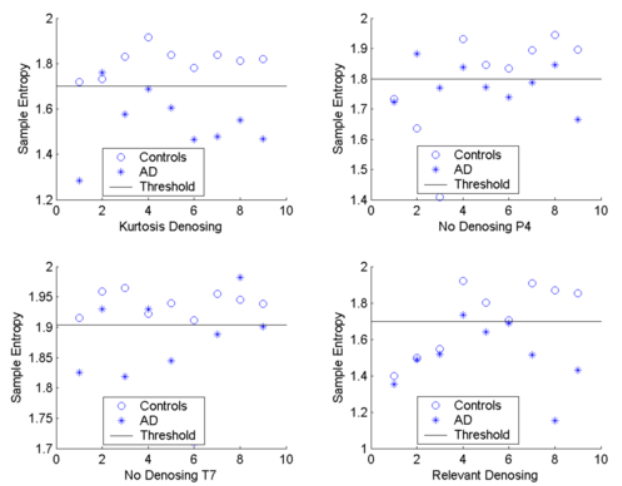

Fig. 2. Threshold classification for the SE values with for the SE values for different methods in Table 7. 
Table 8

Comparison results with ASUME [9] and CSP [27]

\begin{tabular}{lll}
\hline Method & Electrode & Accuracy \\
\hline AMUSE [9] & F3 & $78.7 \%$ \\
CSP [27] & F3 & $75.5 \%$ \\
Kurtosis de-noising & F3 & $94.4 \%$ \\
\hline
\end{tabular}

\section{References}

[1] R. Turner, Biomarkers of Alzheimer's disease and mild cognitive impairment: Are we there yet? Experimental Neurology 183 (2003), 7-10.

[2] C. Chiang, J.H. Steen and C. Chan, A resident's behavior simulation model for nursing home healthcare services, BioMedical Materials and Engineering 24 (2014), 69-75.

[3] F. Fraga, T. Falk, P. Kanda and R. Anghinah, Characterizing Alzheimer's disease severity via resting-awake EEG amplitude modulation analysis, PLoS One, 8 (2013), e72240, doi:10.1371/journal.pone.0072240.

[4] R.M. Chapman, et al., Brain event-related potentials: Diagnosing early-stage Alzheimer's disease, Neurobiology of Aging 28 (2007), 194-201.

[5] D. Ab'asolo, et al., Entropy analysis of the EEG background activity in Alzheimer's disease patients, Physiological Measurement 27 (2006), 241-253.

[6] J. Jeong, EEG dynamics in patients with Alzheimer's disease, Clinical Neurophysiology 115 (2004), 1490-1505.

[7] K. Bennys, G. Rondouin, C. Vergnes and J. Touchon, Diagnostic value of quantitative EEG in Alzheimer's disease, Neurophysiologie Clinique 31 (2001), 153-160.

[8] O.N. Markand, Organic brain syndromes and dementias, in: Current Practice of Clinical Electroencephalography, D.D. Daley and T.A. Pedley, Eds., Raven Press, New York, 1990, pp. 4001-4423.

[9] A. Cichocki, et al., EEG filtering based on blind source separation (BSS) for early detection of Alzheimer's disease, Clinical Neurophysiology 116 (2005), 729-737.

[10] F.B. Vialatte, J. Solé-Casals, M. Maurice, C. Latchoumane, N. Hudson, S. Wimalaratna, J. Jeong and A. Cichocki, Improving the quality of EEG data in patients with Alzheimer's disease using ICA, Lecture Notes in Computer Science 5506 (2009), 979-986.

[11] T. Musha, et al., A new EEG method for estimating cortical neuronal impairment that is sensitive to early stage Alzheimer's disease, Clinical Neurophysiology 113 (2002), 1052-1058.

[12] T. Koenig, et al., Decreased EEG synchronization in Alzheimer's disease and mild cognitive impairment, Neurobiology of Aging 26 (2005), 165-171.

[13] F. Vecchio, et al., Sources and coherence of cortical EEG rhythms could predict progression from mild cognitive impairment to Alzheimer disease, Clinical Neurophysiology 117 (2006), (Suppl. 1) 1-2.

[14] R. Chapman, et al., Brain event-related potentials: diagnosing early-stage Alzheimer's disease, Neurobiology of Aging 28 (2007), 194-201.

[15] K. Van der Hiele, et al., EEG and MRI correlates of mild cognitive impairment and Alzheimer's disease, Neurobiology of Aging 6 (2006), doi:10.1016/j.neurobiolaging.2006.06.006.

[16] F. Vialatte, et al., Blind source separation and sparse bump modeling of time frequency representation of EEG signals: New tools for early detection of Alzheimer's disease, IEEE Workshop on Machine Learning for Signal Processing, $2005,27-32$

[17] S.T. DeKosky and K. Mare, Looking backward to move forward: Early detection of neurodegenerative disorders, Science 302 (2003), 830-834.

[18] J. Dauwels, F. Vialatte, T.M. Rutkowski and A. Cichocki, Measuring neural synchrony by message passing, Proceedings of the Twenty-First Annual Conference on Neural Information Processing Systems, 2007, NIPS2007.

[19] D. Ab'asolo, et al., Analysis of regularity in the EEG background activity of Alzheimer's disease patients with approximate entropy, Clinical Neurophysiology 116 (2005), 1826-1834.

[20] J. Jeong, et al., Nonlinear dynamic analysis of the EEG in patients with Alzheimer's disease and vascular dementia, Clinical Neurophysiology 18 (2001), 58-67.

[21] W. Pritchard, et al., EEG-based, neural-net predictive classification of Alzheimer's disease versus control subjects is augmented by non-linear EEG measures, Electroencephalography and Clinical Neurophysiology 92 (1994), 118-130.

[22] J-H Park, S. Kim, C-H Kim, A. Cichocki and K. Kim, Multiscale entropy analysis of EEG from patients under different pathological conditions, Fractals 15 (2007), 399-404. 
[23] W.L. Woon, A. Cichocki, F. Vialatte and T. Musha, Techniques for early detection of Alzheimer's disease using spontaneous EEG recordings, Physiological Measurements 28 (2007), 335-347.

[24] D.E. Lake, J.S. Richman, M.P. Griffin and J.R. Moorman, Sample entropy analysis of neonatal heart rate variability, American Journal of physiology, Regulatory, Integrative and Comparative Physiology 283 (2002), 789-797.

[25] J.S. Richman and J.R. Moorman, Physiological time series analysis using approximate entropy and sample entropy, American Journal of Physiology, Regulatory, Integrative and Comparative Physiology 278 (2000), 2039-2049.

[26] A. Cichocki and S. Amari, Adaptive Blind Signal and Image Processing: Learning Algorithms and Applications, Wiley, New York, 2003.

[27] H. Ramoser, et al., Optimal spatial filtering of single trial EEG during imagined hand movement, IEEE Transaction on Rehabilitation Engineering 8 (1998), 441-446.

[28] G. Blanchard and B. Blankertz, BCI Competition 2003: Data set: IIa. Spatial patterns of self-controlled brain rhythm modulations, IEEE Transactions on Biomedical Engineering 51 (2004), 1062-1066.

[29] A. Delorme, T. Sejnowski and S. Makeig, Enhanced detection of artifacts in EEG data using higher-order statistics and independent component analysis, Neuroimage 34 (2007), 1443-1449.

[30] A. Hyvärinen and E. Oja, Independent component analysis: Algorithm and applications, Neural Networks 13 (2000), 411-430.

[31] G. Wang, C. Li and L. Dong, Noise estimation using mean square cross prediction error for speech enhancement, IEEE Transactions on Circuits and Systems-I 57 (2010), 1489-1499.

[32] G. Wang, N. Rao, S. Shepherd and C. Beggs, Extraction of desired signal based on AR model with its application to atrial activity estimation in atrial fibrillation, EURASIP Journal on Advances in Signal Processing 2008 (2008), Article ID 728409.

[33] G. Wang, N. Rao and Y. Zhang, Atrial fibrillatory signal estimation using blind source extraction algorithm based on high-order statistics, Science in China, Series F: Information Sciences 51 (2008), 1572-1584.

[34] S. Choi, A. Cichocki and S. Amari, Flexible independent component analysis, Journal of VLSI Signal Processing 26 (2000), 25-38.

[35] J.F. Cardoso and A. Souloumiac, Blind beamforming for non-Gaussian signals, IEE Proceedings F, Radar and Signal Processing 140 (1993), 362-270.

[36] F.D. Wong, L.S. Chao and X.D. Zeng, A supportive attribute-assisted discretization model for medical classification, Bio-Medical Materials and Engineering 24 (2014), 289-295. 